\title{
SIMULATOR-BASED FRAMEWORK TOWARDS IMPROVED CACHE PREDICTABILITY FOR MULTI-CORE AVIONIC SYSTEMS
}

\author{
Jean-Baptiste Lefoul \\ Alexy Torres Aurora Dugo \\ Felipe Magalhaes \\ Gabriela Nicolescu
}

\author{
Dahman Assal \\ Nicolas Ulysse
}

Mannarino Systems \& Software Inc.

100 Boulevard Alexis-Nihon 800

Saint-Laurent, QC H4M 2P4

dahman.assal@mss.ca

nicolas.ulysse@mss.ca

\begin{abstract}
Avionics are highly regulated systems and their criticality requires determinism. Single-core systems have a single processor running, hence only one execution flow, making them easily predictable in execution. However, manufacturers are moving towards multi-core processors manufacturing only. Avionics systems are thus compelled to transition from single-core to multi-core architectures. Mechanisms to mitigate the loss of determinism when using multi-core architectures must be developed to take profit from the parallelism offered by them. This work focuses on partitioned avionics systems and more precisely Real-Time Operating Systems (RTOS) in avionics. We propose a trace-based framework that analyzes application behavior. It also gives cache information through an integrated cache simulator. To validate our framework, we integrated a cache locking selection algorithm. When running tests through our framework and its embedded simulator, we have an improvement in execution predictability and a reduction of interference (over $25 \%$ ) after locking data in the cache.
\end{abstract}

Keywords: Interference mitigation methods, ARINC-653, RTOS, Automation framework, Cache memories.

\section{INTRODUCTION}

Aerospace systems are one of the most critical systems which are open to civilians. As opposed to a failing automobile, which can stop on the side of the road or a failing ship that can be evacuated using rafts, a failing passenger airplane cannot be stopped and evacuated in mid-air. This is the reason why aerospace systems must follow strict certification rules. Aerospace systems are hard real-time systems, which means that they have to follow timing constraints without faulting: every task has a deadline that must be respected. Predictability of such systems is crucial and is a major component of certification for aerospace systems. 
Multi-core architectures are more and more present in embedded systems. Processors manufacturers are slowly stopping single-core production to the profit of more powerful multi-core systems (Geer 2005). Aerospace actors, representing a small part of the consumer processor market need to adapt to keep their hardware up to date. Indeed, aerospace systems cannot rely on multi-core architectures yet: even if providing new capabilities in terms of computing power, it also brings major challenges related to the lack of predictability and safety implications.

Sharing resources in a multi-core system will create so-called interferences between the different cores. (Fuchsen 2010) (De Niz, D. et al. ). From the multi-core designer's viewpoint, interference occurrence isn't a dysfunctional behavior, it's considered a performance bottleneck. However, for the avionics designers, interference occurrences are considered dysfunctional behaviors. Therefore, the failure modes related to interferences and their effects on integrity, availability, or non-deterministic behavior of embedded applications must be identified and mitigated. Moreover, this mitigation has to be achieved while considering the certification additional challenges. One of the best known certification documents on the aerospace domain is the DO-178 guidance (RTCA 2011). Additional avionics standards, such as ARINC-653 (ARINC-653 2015) and CAST-32A (FAA 2016) make it more difficult for multi-core systems to comply with these documents. Current state-of-the-art Real-Time Operating Systems (RTOS) used in avionics (VxWorks (Parkinson 2017), PikeOS (Kaiser, R. and Wagner, S. 2007), Integrity178 (Griglock, M. A. et al. 2012), etc.) are still not certified on multi-core systems. These RTOS tend to force multi-core systems to shut down all additional cores and only execute on the main core to allow certification, resulting in the loss of all advantages brought using multiple processing units (Parkinson 2017).

This work presents a design framework to mitigate cache interferences occurring in multi-core systems, by selecting data to lock in the cache. The contributions of this paper are: $i$ ) a method to trace memory accesses for hardware probes, and ii) the specification and development of a new cache simulator as a module of the framework and an implementation of a cache simulator that supports locking, miss/hit per access and more. When running tests through our framework, we indeed have an improvement in execution predictability and a reduction of interference (over 25\%) after locking data in the cache.

\section{BASIC CONCEPTS}

\subsection{Federated Architecture vs IMA Architecture}

In the end of the twentieth and the beginning of the twenty-first century, avionics systems underwent the transition from federated architectures to Integrated Modular Avionics (IMA) (Watkins, C. B. and Walter, R. 2007). The main reason was to reduce Size, Weight, and Power (SWaP) issues, which are common to most embedded systems.

Figure 1 illustrates the difference between applications implemented with federated architectures and with IMA. In a federated architecture, each application has its own hardware called a Line Replaceable Unit. Line Replaceable Units can be seen as a set of interconnected boxes. The drawback of these easily replaceable hardware units is the cost of redundancy of hardware. This is one of the reasons IMA architectures are used today: one computing unit can be used to support multiple applications, allowing a hardware computing unit to be used by multiple applications, therefore the cost of redundancy is reduced.

\subsection{Cache Memories}

Caches are memory units located between the CPU and the main memory. The cache plays the role of a temporary memory, enabling faster access to frequently used data. Each time a CPU requests memory data, 

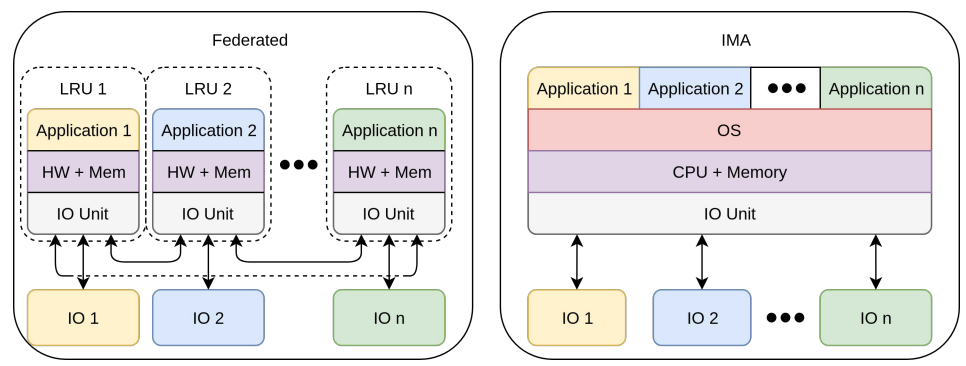

Figure 1: Federated architecture and IMA architecture.

first it requests it from the cache then if the data is not present, the cache requests it from the main memory. The number of CPU cycles required to access data located in the cache is smaller than when the data is located in the main memory. This brings an important increase in performance (faster execution and less power consumption) (Mittal 2014).

Figure 2 illustrates a typical cache architecture in a harvard two-core system. Two (e.g. 16KB) L1 private caches, one for instruction and one for data, and one (e.g. 256KB) L2 shared cache are distributed between the two CPUs. Depending on the access type (instruction or data) and the requesting CPU, the request path will be different throughout the cache levels. For instance, if CPU 0 requests an instruction, its first private level of instruction cache (L1I) is checked. If the instruction is not there, then L2 is checked. If the instruction cannot be accessed then the main memory is checked.

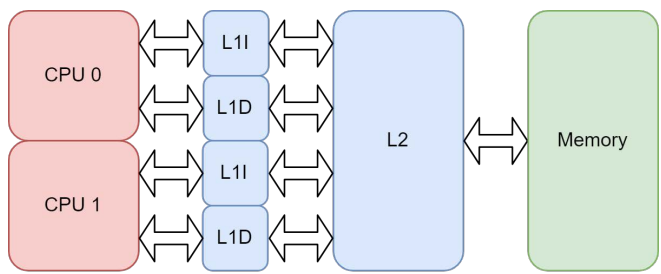

Figure 2: Common cache architecture in dual core systems.

\subsection{Interferences in Shared Resources}

When parallel applications are trying to access the same resource, only one core can be given the access, which impacts the performance of the others. This highlights the fact that one application can have unintended impact on the execution of another one. This phenomenon is called interference.

In (Fuchsen 2010), several interference channels are discussed, either software or hardware. The channels that impact the most the execution determinism are those related to the caches (Fuchsen 2010) (Huyck 2012). The most important issue with caches is that one application may evict cache data used by another application. This is an indirect interference. Another type of interference is the contention on a shared resource. For instance, if in a multi-core system, one CPU requests access to one resource, which is being used by another CPU, this impacts the performance. This is a direct interference.

Interferences have several drawbacks, one of them being that, depending on the second application to run in parallel, the execution time of a given interfered application is increased. This brings non-deterministic behavior of the application, which cannot be tolerated in avionics systems where lives depend on the correct execution of the system. 


\subsection{Partitioned Multi-Core RTOS: ARINC-653}

ARINC-653 (ARINC-653 2015) is a standard that gives specifications for partitioned RTOS. According to this standard, the partitioning must be done in space and in time.

Space Partitioning Each partition is isolated regarding hardware usage, such as memory space: each partition has a set of addresses in memory and is the only one having the rights to access them

Time Partitioning The CPU time is divided in several time windows. Each time window is allocated to a partition. During one of its time windows, a partition is the only one executing on a CPU. All partitions are allocated time windows within a period of time called major time frame. The schedule is repeated every major time frame.

The standard also specifies services that the RTOS must offer. These services are called APEX services. As this is not the scope of this work, the APEX services will not be covered.

\section{STATE-OF-THE-ART}

Different types of interference might occur in the system: shared memory, interconnects and I/O, for instance. This work focuses on the interference in the cache level. Mitigation solutions for cache interference, as well as cache profiling and cache simulation are thus discussed.

A common approach for assessing hardware execution of a system, in order to analyze the interference in the cache, is to use a cache simulator. Cache simulators can be used to gather information on the behavior of a cache. Such information is the number of misses per cache level, which gives a hint on the contention in the cache. Plenty of cache simulators are already proposed in the literature, but most of them are for educational purpose and/or only support simple cache replacement policies ( Yuan, X. et al. 2004). A generic cache simulator that can be configured to simulate all types of cache architecture is required to explore the cachelevel interference mitigation solutions. Moreover, since we want to simulate the cache locking, we require specifically a simulator that models this technique.

SMPCache Described in (Vega Rodríguez, M. A. et al. 2001), the cache simulator SMPCache is a graphical educational trace-based cache simulator. We can execute the traces step by step and see the evolution of the cache characteristics such as what data is loaded and whether the access was a cache miss or hit. Unfortunately, SMPCache does not support cache locking, but only instruction fetch, data read and write. The traces used for SMPCache holds three fields in an ASCII file: the requesting processor, the access type (instruction fetch, data read or data write) and the accessed address. One can configure the cache (replacement policy, cache coherency protocol, etc.).

Dinero IV Dinero IV (Edler and Hill 1998) is a single-core cache simulator. The simulator allows the user to configure the cache architecture to be simulated according to a set of parameters: cache levels, cache size, block size, replacement policy, associativity. The obtained simulation results consist of the amount of cache hits/misses per cache and per access type (instruction or data). In addition to instruction fetches, the number of data reads and misses, Dinero simulates cache invalidation instructions.

None of the above simulators take into account the cache locking approach. This is a key feature for the exploration of cache locking algorithms. In order to enable this exploration, we propose a new simulator. Table 1 gives an overview of the main requirements for a cache simulator to be used for interference mitigation solutions exploration. We also position our contribution according to these required features. 
Table 1: Solutions comparison.

\begin{tabular}{|c|c|c|c|}
\cline { 2 - 4 } \multicolumn{1}{c|}{} & SMPCache & Dinero & Our cache simulator \\
\hline $\begin{array}{c}\text { Cache architecture configuration } \\
\text { (cache levels, size, etc.) }\end{array}$ & $\checkmark$ & $\checkmark$ & $\checkmark$ \\
\hline Step by step simulation & $\checkmark$ & $\checkmark$ & $\checkmark$ \\
\hline Use as a library & X & $\checkmark$ & $\checkmark$ \\
\hline $\begin{array}{c}\text { Memory footprint optimization } \\
\text { (binary trace file) }\end{array}$ & X & $\checkmark$ & $\checkmark$ \\
\hline $\begin{array}{c}\text { Acceptable execution time } \\
\text { (<1 min / 100k accesses) }\end{array}$ & X & $\checkmark$ & $\checkmark$ \\
\hline Custom replacement policy & X & $\checkmark$ & $\checkmark$ \\
\hline Cache locking simulation & X & X & $\checkmark$ \\
\hline
\end{tabular}

Acknowledging that the goal of this work is on the cache profiling and simulation, we have integrated a cache locking solution in order to validate our solution. We present next a review on the cache mitigation mechanisms.

In (Mancuso, R. et al. 2013), the authors propose a framework that profiles memory access of a task in order to get the access pattern of the task. Once the pattern is obtained, an offline algorithm is responsible for computing the memory mapping of virtual addresses to perform a cache division technique (i.e., cache coloring). Additionally, the algorithm selects cache lines to lock for better predictability. This differs from our contribution by locking only the last level cache.

In (Qureshi, M. K. and Patt, Y. N. 2006), a runtime mitigation of interferences on the cache is proposed. Additional hardware is added to the system which monitors accesses to each way of the cache. A partitioning algorithm then computes, with the information gathered by the added hardware, the way-based cache partitioning for the system's application. The cache replacement policy is also modified to take into account the computed partitions.

Many architectures provide support for cache management, allowing designers to manipulate the cache blocks at will. The cache locking technique is used to lock in the cache the data that are not subject to eviction. This way, if the data is requested, it will always hit in the cache. The challenge employing this technique is to properly select the data to lock as well as when to lock this data (Mittal 2016). To lock the cache, one can rely on two different approaches: static locking (Liang, Y. and Mitra, T. 2010) or dynamic locking (Vera, X. et al. 2003). The static technique locks all the data in the cache before the system starts to execute. With this technique, the locked contents of the cache cannot be changed while the system is running. Dynamic locking works during system execution. It employs different algorithms to choose which lines to lock and unlock during execution time, relying on a different set of criteria.

A variety of approaches are employed when selecting the type of data to be locked. Some contributions selected to fully lock (Zheng, W. et al. 2017), which renders cache deterministic because either a datum is locked and each request to it hits in the cache or it is not locked and each request is a miss. To cope with this limitation, most works practice the partial cache locking (Loach, M. and Zhang, W. 2015), where the cache is not fully locked or not locked at all.

To select the contents to lock in the cache, one can statically analyze the application (Ding, H. et al. 2012) or dynamically analyze the application (Adegbija, T. and Gordon-Ross, A. 2018). The static analysis is done by analyzing the application code without running it. Dynamic analysis is done by profiling the application and select data to lock in the cache based on the information gathered during the application's execution.

For avionic systems, at every partition switch, the cache contents are flushed. This way, the cache locking configuration has to be reloaded each partition switch. Hence, compared with the above-mentioned works, the algorithm we use relies in pseudo-static partial locking of both instruction and data of both user and 
kernel spaces (Torres, A. et al. 2019). It takes cache misses and hits into account using dynamic analysis and a cache simulator.

\section{CACHE LOCKING FRAMEWORK}

The developed framework consists of a set of inputs, outputs and the relation between them. Each aspect will be presented separately, for the sake of understanding.

\subsection{Inputs and Outputs Relationship}

The overview of the proposed framework is given in Figure 3. It is worth noting that the targeted system architecture must support locking and that the RTOS used supplies a locking API. The framework is divided into three main modules: the memory access tracer, the cache simulator and the cache locking algorithm. Each module can be replaced by another version, as long as the inputs/outputs are respected.

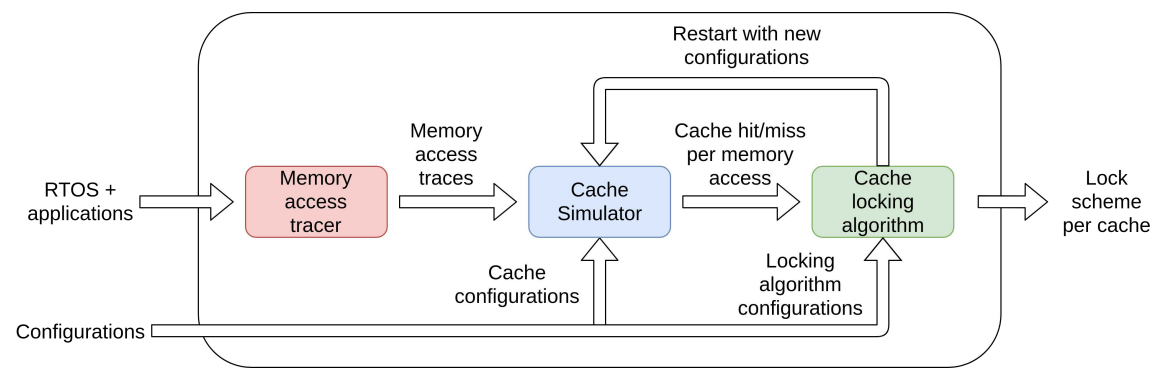

Figure 3: Cache locking framework overview.

\subsubsection{Memory Access Tracer}

The role of the memory access tracer is to gather traces from the execution of applications on an RTOS. The memory tracer is also in charge of storing traces in a usable format for the cache simulator and the cache locking algorithm. The tracer is based on a well-defined trace structure that we proposed. We propose two approaches for the tracer implementation: (1) a QEMU-based approached and (2) a hardware probe approach.

QeMU QEMU is the most industrially used open-source system emulator that supports a broad range of architectures (Bellard 2005). An emulator behaves as the system behaves but does not represent how the system achieves this behavior.

In order to collect the traces, we had to instrument QEMU. This instrumentation enabled us to create the traces and activate/deactivate the tracing when required. To create the traces, each time an instruction is emulated within QEMU, we check if it's an instruction required to trace, then we create a trace.

After collecting the traces from QEMU, we need to activate or deactivate the tracing using two instructions: one for the activation and one for the deactivation. We propose a solution that takes advantage of the instructions that are not used in the emulated architectures. In the case of the PowerPC e500 processor, used as a test platform, $0 x F F F F F F F 1$ and $0 x F F F F F F F 0$ are two unused instructions.

Exploiting QEMU for tracing brings the advantage of flexibility and facility to trace memory accesses for new architectures. However the emulation time is slow compared with the hardware execution. Moreover, the timing behavior, one of the main dimensions of the ARINC 653 RTOS, may not be considered. 
Hardware Probe A hardware probe is a system capable of gathering information on the system while it executes without modifying the system's behavior. It is very important to use non-intrusive hardware probes. The probe we used is the LA-7630 NEXUS Debug/Trace probe from Lauterbach.

To obtain traces using a hardware probe, a specific software is required. In our case, we obtained traces using the NEXUS probe which requires the Trace 32 software. The software can be used to trace a given section of a program (defined by two points in the program). The traces are exported in a binary. A translation of this binary to a binary compatible with our framework was required and thus developed. The debugging software allowed us to set the beginning and the end of tracing in the program, which made the tracing easier.

\subsubsection{Cache Simulator}

The second module is responsible for a step-by-step simulation of the behavior of the cache when executing memory access traces provided by the first module. This module is covered in detail in Section 5.

\subsubsection{Cache Locking Algorithm}

The third module is responsible for selecting the addresses of data to lock in the cache, using the results obtained with the cache simulator. In order to do so, the user can specify for each cache the latency of a hit as well as the latency of a miss. When running the cache simulator, we can obtain the information on each operation performed on the cache.

$$
\begin{aligned}
& \text { Cost }_{A d d r, C_{i}}=\text { hits }_{\text {Addr }, C_{i}} \times \text { hitLatency }_{C_{i}}+\text { misses }_{\text {Addr }, C_{i}} \times \text { missLatency }_{C_{i}} \text {, } \\
& \operatorname{Cost}_{\text {Addr }}=\sum_{C_{i}}\left(\operatorname{Cost}_{\text {Addr, }}\right) \text {, } \\
& \operatorname{Cost}_{S e t_{i, C_{j}}}=\sum_{A d d r \in \operatorname{Set}_{i, C_{j}}}\left(\operatorname{Cost}_{A d d r, C_{j}}\right), \\
& \operatorname{Cost}_{C_{i}}=\sum_{\operatorname{Set}_{j, C_{i}}}\left(\operatorname{Cost}_{\operatorname{Set}_{j, C_{i}}}\right), \\
& \text { TotalCost }=\sum_{C_{i}}\left(\operatorname{Cost}_{C_{i}}\right) \text {, }
\end{aligned}
$$

where $C_{i}$ is the cache of index $i, \operatorname{Set}_{i, C_{j}}$ is the set of addresses of the $i^{\text {th }}$ set of cache $C_{j}$, hit Latency $C_{C_{i}}$ (resp. missLatency $C_{i}$ ) is the latency to manage a cache hit (resp. a cache miss) in the cache $C_{i}$, hit $s_{A d d r, C_{i}}$ (resp. misses $_{A d d r, C_{i}}$ ) is the number of cache hits (resp. cache misses) at address $A d d r$ in cache $C_{i}, \operatorname{Cost}_{A d d r, C_{i}}$ is the cost induced by the hits and misses of address $A d d r$ in the cache $C_{i}, \operatorname{Cost}_{A d d r}$ is the cost induced by the hits and misses at address Addr, Cost $_{\text {Set }_{i, C_{j}}}$ is the cost induced by the hits and misses in $\operatorname{Set}_{i, C_{j}}$ and TotalCost is the total cost of a cache configuration for a certain trace.

\subsection{Framework Input}

The framework has two sets of inputs : the first set concerns the cache architecture while the second set is related to the applications for which the cache locking selections must be done.

The cache-related inputs are: 
- Cache configurations: the configuration is defined by the cache index and its own configuration given by its size, the number of ways, the number of sets and the replacement policy. The cache configuration is defined in the expression (6).

CacheConf $=\{($ cacheId, conf $) \mid$ cacheId $\in \llbracket 0, N-1 \rrbracket\}$,

where $N$ is the number of caches, cacheId is a cache index and conf the configuration of the cache referred by cacheId.

- CPU cache entry points: each CPU has two entry points to the cache (in Harvard architectures). CoreCacheMap represents the correspondence of the caches with their entry points. CoreCacheMap is defined in the expression (7).

CoreCacheMap $=\left\{\left(\right.\right.$ cpuId,$\left(\right.$ cache $_{\text {inst }}$, cache $\left.\left._{\text {data }}\right)\right) \mid$ cpuId $\in \llbracket[0, M-1 \rrbracket\}$,

where $M$ is the number of CPUs, cache $_{\text {inst }}$ (resp. cache data $_{\text {) }}$ ) represent the index of the instruction entry point (resp. the data entry point) for the CPU of index cpuId.

- Cache-level relationship: each cache is related to a lower-level cache, except for the last level caches for which the next level is the main memory. This relationship is defined in the expression (8).

$$
\text { NextLvlCacheMap }=\{(\text { cacheId, } \text { nextCacheId }) \mid \text { cacheId } \in[0, N-1 \rrbracket\},
$$

where $N$ is the number of caches and nextCacheId is the index of the next level cache of the cache with the index cacheId.

The applications' related inputs are:

- The binary of the RTOS and of the applications. We will use the notation Binary for this type of inputs.

- The ARINC-653 schedule of the partitions:

Schedule $=\operatorname{array}(($ offset, partitionId $))$,

a sorted array representing the time windows in the major time frame where offset is the offset in the major time frame of the partition of index partititonId.

The input set of the defined framework is defined by the expression (10).

$$
\text { input }=(\text { CacheConf }, \text { CoreCacheMap, NextLvlCacheMap }, \text { Binary, Schedule }) \text {. }
$$

\subsection{Framework Outputs}

The framework's output is given by a set of addresses to lock in each cache and for each application. The output is defined in the expression (11).

$$
\begin{aligned}
\text { out put }= & \{(\text { partitionId }, \text { cacheId }, \text { addressList }) \mid \\
& \text { partitionId } \in \llbracket[0, K-1 \rrbracket \text { and cacheId } \in \llbracket[0, N-1 \rrbracket\},
\end{aligned}
$$

where addressList is a list of addresses to lock in the cache of index cacheId for the partition of index partitionId, $N$ is the number of caches and $K$ is the number of partitions.

It is to be noted that the selected lines to lock are specific to the applications and the RTOS given as inputs and they are obtained considering the execution determinism of the applications in the system. 


\section{CACHE SIMULATOR}

The cache simulator is used to give information on the CPU accesses to the cache. Hence, the simulator models the behavior of the cache in order to detect the access misses or hits in the cache. The base components of the cache simulator are the cache line, the cache set, the whole cache and the cache address.

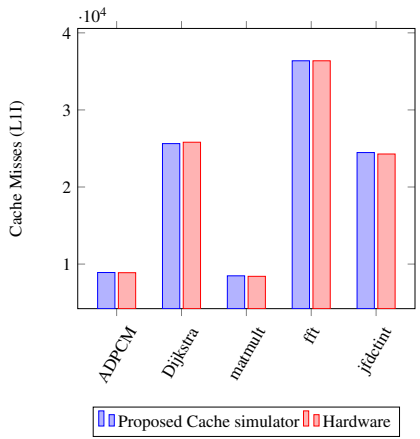

(a)

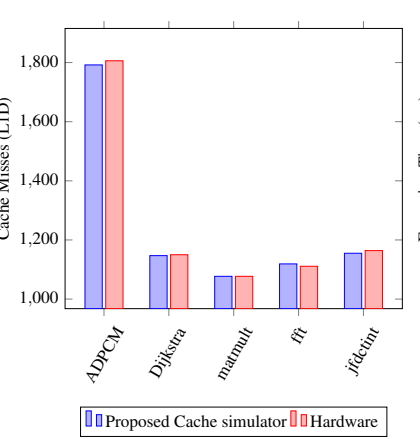

(b)

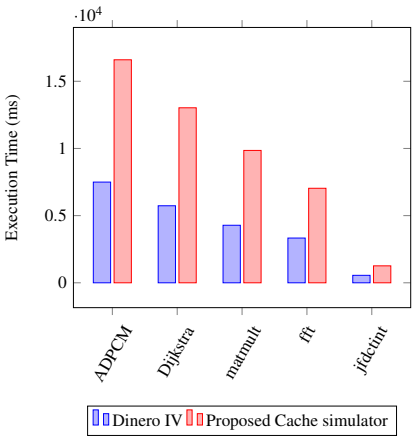

(c)

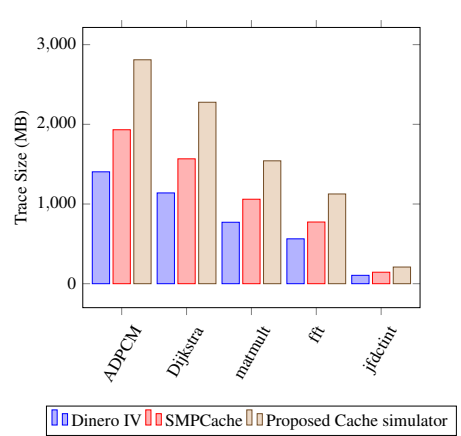

(d)

Figure 4: Number of cache misses: (a) L1D and (b) L1I (c) Execution time in ms and (d) Trace file size in MB.

Cache Line A cache line (or cache block) is a block of data repatriated from the memory. Data are generally not repatriated byte per byte from the memory, but by chunks of bytes.

Cache Set A cache set contains one or more cache lines, depending on the associativity of the cache. There are three types of associativity: direct-mapped cache (1 way-associative), fully associative (only one set, the number of ways is the number of cache lines) and set associative (n-way associative). These three types can be represented by an n-way associativity with $n$ between 1 (direct mapped) and the number of lines in the cache (fully associative).

Cache A cache contains one (in the case of a fully associative cache) or more cache sets. There can be several layers of caches between the CPU and the main memory. Each cache is either connected to one next level cache or to the main memory (which means the cache is the Last Level Cache).

Replacement Policy The replacement policy is responsible for choosing which line in the cache set must be evicted when a requested address's tag does not match the tag of one of them. The perfect replacement policy would be to evict the cache line which data will be accessed last compared to the other cache lines (Belady 1966). However, this replacement policy requires knowledge of the future accesses to the cache set, rendering it unfeasible. We implemented in our simulator three of the most popular replacement policies: Pseudo Least Recently Used, First-In-First-Out and Pseudo Round Robin.

The cache simulator allows the user to configure the memory cache hierarchy according to the following parameters: $i$ ) cache architecture (the number of cache levels, how they are related, the core affinity of each cache); ii) cache size and organization (associativity, size of cache line); iii) address size and representation (Physically or virtually tagged and indexed); iv) replacement policy (PLRU, FIFO Pseudo Round Robin), and; $v$ ) other hardware-specific information can be added (such as whether a flush invalidates the cache). 


\section{RESULTS}

Presented results seek to show the cache simulator accuracy as well as the reduction in cache misses when using our framework. Results were obtained using benchmark applications from the suites Mibench and SNURT: such as ADPCM, matrix multiplication and Dijkstra's algorithm.

\subsection{Simulator Validation With Respect to Hardware}

We modeled in our simulator the cache configuration implemented in the e200z7 PowerPC architecture. Before the tracing, the cache must be flushed and invalidated in order to be in synchronization with the simulator which starts with all invalidated cache lines. Figures 4.(a) and 4.(b) regroups the obtained results. As it is possible to see, the obtained readings are close to the real reading, from probing hardware. In fact, the error rate is below $0.8 \%$.

\subsection{Simulator Validation With Respect to Other Cache Simulators}

We compared the framework's cache simulator with the ones from the state of the art (Dinero IV (Edler and Hill 1998) and SMPCache (Vega Rodríguez, M. A. et al. 2001)). The number of cache misses and hits obtained from the three solutions is similar, with a slightly higher accuracy for our solutions (4\%). Figures 4.(c) and 4.(d) show the performance in execution time and the size of the required traces for the Dinero IV and the proposed simulator. The obtained results reflect the correlation between the size of the trace file and the execution time of the simulators. We could not show the execution time of SMPCache, because it was longer than a day of execution for ADPCM. This is mainly due to the fact that SMPCache not only simulates the caches but also other memory-related mechanisms.

The proposed cache simulator takes about twice as long to execute than Dinero IV. This is due to the fact that the framework's simulator gives more information and simulates more than instruction fetches, data reads and data writes. The trace size is two times bigger but the traces contain more information.

\subsection{Impact on Execution}

To assess the impact on the execution, the test benches were modified to request a high amount of data, which would mean a high number of accesses to the cache. This was done by increasing the sizes of the data used in each benchmark. We executed two applications concurrently on a dual-core system (one on each CPU) with the following pairing: (1) ADPCM / Dijkstra, (2) FFT / Dijkstra, (3) SHA1 (encryption) / JFDCT and (4) QSORT / ADPCM. As illustrated in Figure 5, we have reduction in L2 cache requests and in inter-core cache evictions. This shows that our solution can potentially reduce cache-related interferences (direct and indirect) in multi-core systems.

\section{CONCLUSION}

With the evolution of multi-core architectures today and the need of aerospace industry to use commercially available Off-The-Shelf (COTS) processors, system developers in this industry must adapt and be ready to use Muliprocessor System-on-Chip (MPSoC). Unfortunately, multiprocessing brings multiple challenges, including interferences between running applications caused by parallel execution of applications using common hardware resources. It is therefore imperative to mitigate these interferences in order to comply with aerospace certifications and standards. This paper presents a framework that gives tools to develop/implement solutions to mitigate cache interferences. It is capable of reducing over $25 \%$ of interferences in 


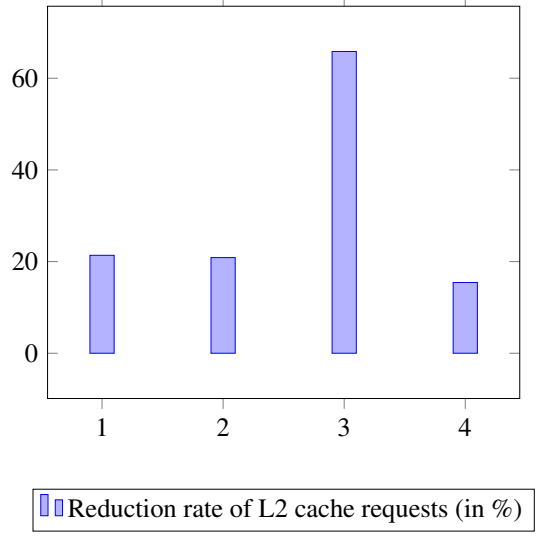

(a)

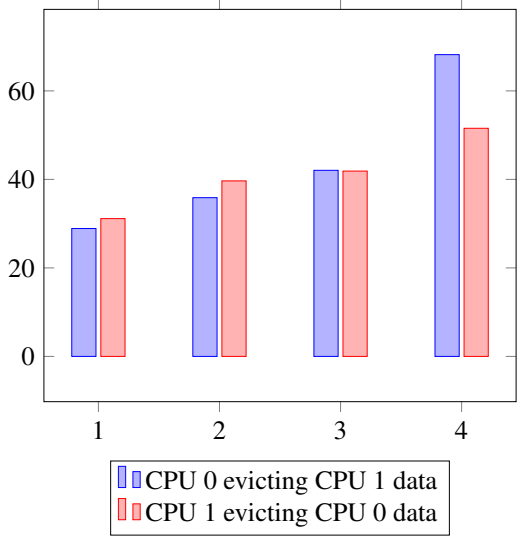

(b)

Figure 5: Experimental results in multi-core architectures. (a) Reduction rates for the number of cache requests in $\mathrm{L} 2$ cache and (b) reduction rate for cache block data evicted between the two CPUs.

the cache by reducing the number of cache misses in private caches using a cache locking approach. The mitigation can also be observed on the standard deviation of execution time (more than $60 \%$ improvement) highlighting the increase of determinism brought by our solution.

\section{REFERENCES}

Yuan, X. et al. 2004, March. "Cache Memory Simulators: A Comparative Study". In IEEE SoutheastCon, 2004. Proceedings.

Adegbija, T. and Gordon-Ross, A. 2018, Jan. "PhLock: A Cache Energy Saving Technique Using PhaseBased Cache Locking". IEEE Transactions on Very Large Scale Integration (VLSI) Systems vol. 26.

ARINC-653 2015, Aug. "ARINC 653P0-1 Avionics Application Software Standard Interface, Part 0, Overview of ARINC 653".

Belady, L. A. 1966, June. "A Study of Replacement Algorithms for a Virtual-storage Computer”. IBM Syst. J. vol. 5 (2).

Bellard, F. 2005. "QEMU, a Fast and Portable Dynamic Translator". In Proceedings of the Annual Conference on USENIX Annual Technical Conference, ATEC '05, USENIX Association.

De Niz, D. et al. COTS Multicore Processors in Avionics Systems: Challenges and Solutions.

Ding, H. et al. 2012, June. "WCET-centric partial instruction cache locking". In DAC Design Automation Conference 2012.

Edler, J. and Hill, M. D. 1998. "Dinero IV Trace-Driven Uniprocessor Cache Simulator".

Federal Aviation Administration - FAA 2016, Nov. "CAST 32A - Multi-core Processors".

Fuchsen, R. 2010, Oct. "How to address certification for multi-core based IMA platforms: Current status and potential solutions". In 29th Digital Avionics Systems Conference.

Geer, D. 2005, May. "Chip makers turn to multicore processors”. Computer vol. 38 (5), pp. 11-13.

Griglock, M. A. et al. 2012, Nov. "Time-variant scheduling of affinity groups on a multi-core processor".

Huyck, P. 2012, Oct. "ARINC 653 and multi-core microprocessors - Considerations and potential impacts". In 2012 IEEE/AIAA 31st Digital Avionics Systems Conference (DASC). 
Kaiser, R. and Wagner, S. 2007, 02. "Evolution of the PikeOS Microkernel".

Liang, Y. and Mitra, T. 2010, June. "Instruction cache locking using temporal reuse profile". In Design Automation Conference.

Loach, M. and Zhang, W. 2015, April. "Exploring hybrid cache locking to balance performance and time predictability". In SoutheastCon 2015.

Mancuso, R. et al. 2013, April. "Real-time cache management framework for multi-core architectures". In 2013 IEEE 19th Real-Time and Embedded Technology and Applications Symposium (RTAS).

Mittal, S. 2014. "A survey of architectural techniques for improving cache power efficiency". Sustainable Computing: Informatics and Systems vol. 4 (1), pp. 33 - 43.

Mittal, S. 2016, 05. "A Survey Of Techniques for Cache Locking”. ACM Transactions on Design Automation of Electronic Systems vol. 21.

Parkinson, P. 2017, 04. "Update on using multicore processors with a commercial ARINC 653 implementation".

Qureshi, M. K. and Patt, Y. N. 2006, Dec. "Utility-Based Cache Partitioning: A Low-Overhead, HighPerformance, Runtime Mechanism to Partition Shared Caches". In 2006 39th Annual IEEE/ACM International Symposium on Microarchitecture (MICRO'06).

RTCA 2011, Dec. "DO-178C - Software Considerations in Airborne Systems and Equipment Certification".

Torres, A. et al. 2019, 10. "Cache Locking Content Selection Algorithms for ARINC-653 Compliant RTOS". ACM Transactions on Embedded Computing Systems vol. 18, pp. 1-20.

Vega Rodríguez, M. A. et al. 2001. "An educational tool for testing caches on symmetric multiprocessors". Microprocessors and Microsystems vol. 25 (4).

Vera, X. et al. 2003, June. "Data Cache Locking for Higher Program Predictability". SIGMETRICS Perform. Eval. Rev. vol. 31 (1).

Watkins, C. B. and Walter, R. 2007, Oct. "Transitioning from federated avionics architectures to Integrated Modular Avionics". In 2007 IEEE/AIAA 26th Digital Avionics Systems Conference.

Zheng, W. et al. 2017, March. "WCET-Aware Dynamic I-Cache Locking for a Single Task". ACM Trans. Archit. Code Optim. vol. 14 (1).

\section{AUTHOR BIOGRAPHIES}

JEAN-BAPTISTE LEFOUL is a PhD student at Ecole Polytechnique de Montréal. His email is jeanbaptiste.lefoul@polymtl.ca.

ALEXY TORRES is a PhD student at Ecole Polytechnique de Montréal. His email is alexy.torres-auroradugo@polymtl.ca.

FELIPE MAGALHAES is a postdoctoral fellow at Ecole Polytechnique de Montréal. His email is felipe.gohring-de-magalhaes@polymtl.ca.

DAHMAN ASSAL is a Senior Software Designer / Technical Specialist at Mannarino Systems \& Software Inc. in Saint-Laurent, Québec, Canada. His email is dahman.assal@mss.ca.

NICOLAS ULYSSE is a Software Engineering Chief / Engineering Project Manager at Mannarino Systems \& Software Inc. in Saint-Laurent, Québec, Canada. His email is nicolas.ulysse@mss.ca.

GABRIELA NICOLESCU is a Professor at Ecole Polytechnique de Montréal. Her email is gabriela.nicolescu@polymtl.ca. 